\title{
4 THE ACPA IGM FINE SPECIFICITY DIFFERS FROM THE ACPA IGG ANTIGEN-RECOGNITION PROFILE
}

A Willemze, 'P Suwannalai, ' L van Toorn, ${ }^{1}$ G Stoeken-Rijsbergen, ${ }^{1}$ EW N Levarht, ${ }^{1} \mathrm{~J}$ W Drijfhout, ${ }^{2}$ T W J Huizinga, ${ }^{1}$ R E M Toes, ${ }^{1}$ L A Trouw ${ }^{1}$ 'Department of Rheumatology, Leiden University Medical Center, Leiden, The Netherlands; ${ }^{2}$ Department of Immunohematology and Blood tranfusion, Leiden University Medical Center, Leiden, The Netherlands

10.1136/annrheumdis-2011-201234.4

Background The antigen-recognition pattern of immunoglobulin $\mathrm{M}$ ( $\operatorname{Ig} M)$ could, in case directed against protein-antigens, provide an indication of the antigenic moieties triggering new $\mathrm{B}$ cells. The half-life of IgM is short and memory B cells against $T$ cell dependent protein antigens, typically produce IgG and not IgM antibodies. Here the authors analysed whether a difference exists between the fine specificity of IgM versus IgG anticitrullinated protein antibodies (ACPA).

Materials and methods The authors determined the fine specificity of ACPA IgM and IgG in 138 ACPA positive rheumatoid arthritis (RA) patients. Fine specificity assays were performed by ELISA, using citrullinated peptides derived from, vimentin, fibrinogen- $\beta$, fibrinogen- $\alpha$ and $\alpha$-enolase, as well as citrullinated protein antigens, fibrinogen and myelin basic protein. The arginine counterparts were used as control.

Results Recognition of defined citrullinated antigens by IgM ACPA was confined to samples that also display recognition by IgG ACPA. However, the IgM ACPA response displayed a more restricted antigen recognition profile as compared to $\operatorname{IgG}$ ACPA (OR 0.26, $\mathrm{p}<0.0001$ ).

Conclusion Our data show that the immune response against several defined citrullinated antigens varies as some are only recognised by IgG ACPA and others also by IgM ACPA. The recognition of some but not other citrullinated antigens by IgM ACPA suggest that not all citrullinated antigens activate new $B$ cells despite concurrent recognition by IgG ACPA. 